\title{
What causes auditory distraction?
}

\author{
William J. Macken, Fiona G. Phelps, and Dylan M. Jones \\ Cardiff University, Cardiff, Wales
}

\begin{abstract}
The role of separating task-relevant from task-irrelevant aspects of the environment is typically assigned to the executive functioning of working memory. However, pervasive aspects of auditory distraction have been shown to be unrelated to working memory capacity in a range of studies of individual differences. We measured individual differences in global pattern matching and deliberate recoding of auditory sequences, and showed that, although deliberate processing was related to short-term memory performance, it did not predict the extent to which that performance was disrupted by task-irrelevant sound. Individual differences in global sequence processing were, however, positively related to the degree to which auditory distraction occurred. We argue that much auditory distraction, rather than being a negative function of working memory capacity, is in fact a positive function of the acuity of obligatory auditory processing.
\end{abstract}

Although the visual world may be excluded by averting or closing one's eyes or turning one's head, the auditory organ comes equipped with no analogous mechanical means of excluding unwanted physical stimulation; as such, the auditory world remains sensuously ineluctable. That the ears are ever open confers certain benefits whereby adaptively relevant, auditorily signified events that are outside the immediate purview of the animal's engagement need not go unregistered. However, a by-product of ongoing registration of the acoustic environment is that currently goalirrelevant information impinges on the organism and may engage obligatory processing to the potential detriment of any focal task (Hughes \& Jones, 2003a).

One demonstration of the cost of obligatorily processing auditory information is found in what we term the irrelevant sound effect. This refers to the substantial impairment in short-term memory performance that occurs when participants are exposed to task-irrelevant sound (e.g., Banbury \& Berry, 1998; Colle \& Welsh, 1976; Ellermeier \& Zimmer, 1997; Jones \& Macken, 1993; Salamé \& Baddeley, 1982). In a typical experiment, participants are presented with a sequence of six to nine items (e.g., words, digits, and consonants), at a rate of about $1 \mathrm{item} /$ sec; their task is to recall the list in its original order. On some trials, background sound is presented that participants are instructed to ignore and the contents of which they are never queried about. Nonetheless, such background sound produces substantial and ongoing disruption of performance. There are a number of perhaps surprising aspects to the pattern of this distraction. For example, the degree of disruption does not diminish over time, either within an experimental episode or across many days (e.g., Hellbrück, Namba, \& Kuwano, 1996; Jones, Macken, \& Mosdell, 1997), nor does it depend on the intensity of the sound (e.g., Colle \& Welsh, 1976), nor indeed on the pre- cise content of the sound, with sounds as diverse as pure tones, music, narrative speech, and band-pass noise bursts producing a functionally equivalent pattern of disruption (e.g., Jones \& Macken, 1993). Rather, the critical characteristic of the sound that confers its disruptive potency is what we refer to as changing state: The sound must be segmentable into perceptually discrete entities (tones, syllables, etc.), and each element must differ from the one that preceded it. For example, a series of discrete tone bursts changing in frequency causes disruption, whereas a series of tone bursts repeated at the same frequency does not. Similarly, a continuous tone glide, even one containing substantial frequency modulation, does not interfere, whereas an equivalent tone glide, segmented by silent interrupts, does disrupt short-term memory performance.

Furthermore, that aspect of performance susceptible to disruption by task-irrelevant sequences is specific to the short-term retention of sequential information: Memory for items is unimpaired, but memory for the order of those items is disrupted (e.g., Beaman \& Jones, 1997; Jones \& Macken, 1993). For example, if participants are presented one at a time with a randomly ordered sequence of 6 of the 7 days of the week and required to identify the missing day, the usual disruption is not observed; although each item must be retained through the trial, the order in which items are presented is irrelevant. However, if - given the same presentation conditions - participants are now required to say which day was presented after a given day in a particular random ordering, the usual disruption is observed (Jones \& Macken, 1993). This symmetry between the presence of sequences of sounds in the auditory, irrelevant stream, and the requirement to retain sequential information in the focal sequence led us to propose a pattern of distraction based on interference due to similarity of process; the obligatory processing of ordered elements 
within the irrelevant sequence interferes with the ability to deliberately process order within the memory sequence.

Important for our present concern is that there exist considerable individual differences in the susceptibility to interference from irrelevant sound. For example, Ellermeier and Zimmer (1997) found that although typical performance decrement in the presence of background sound was on the order of $50 \%$, the degree of disruption across participants ranged from about $300 \%$ poorer performance in noise, relative to quiet, to a slight enhancement in the presence of noise for some participants. Furthermore, these individual differences are stable over time (Ellermeier \& Zimmer, 1997) and different types of background sound (e.g., speech and nonspeech) (Elliott \& Cowan, 2005). As such, susceptibility to the type of distraction represented by the irrelevant sound effect would appear to reflect genuine quantitative individual differences in cognitive processing. Despite this, it is not clear what aspect of processing underlies these differences. Indeed, many mechanisms that might suggest themselves turn out not to account for these individual differences in susceptibility to irrelevant background sound.

Typically, the ability to focus on task-relevant, and exclude task-irrelevant, information is ascribed to the executive functions of working memory (e.g., Baddeley, 1990; Cowan, 1995; Lavie, 2005; Shiffrin \& Schneider, 1977). Working memory capacity is positively related to a range of higher order cognitive functions, including language comprehension, reasoning, and fluid intelligence (e.g., Daneman \& Carpenter, 1980; Engle, Tuholski, Laughlin, \& Conway, 1999; Süß, Oberauer, Wittman, Wilhelm, \& Schulze, 2002). Critically, it has been argued that working memory capacity affects the extent to which individuals are able to exercise attentional control, particularly in settings where sources of information competing with the task-relevant source need to be inhibited (e.g., Engle, Kane, \& Tuholski, 1999). We might therefore expect a relationship between an individual's working memory capacity and the extent to which he or she is able to inhibit processing of task-irrelevant information. Indeed, there is evidence linking working memory capacity to the ability to exclude task-irrelevant auditory information. Conway, Cowan, and Bunting (2001) found a negative relationship between working memory capacity and the tendency to notice salient information in the unattended ear during a dichotic shadowing task. Conway et al. suggested that this reflected the weaker inhibitory control in those participants with lower working memory capacity, enabling aspects of the task-irrelevant source to "leak" into processing. However, although working memory capacity-whether conceived of as pure storage capacity or as executive processing capacity - might provide a plausible mechanism underpinning the individual differences in susceptibility to the effects of irrelevant sound, several attempts have failed to find any correlation between an individual's simple (e.g., digit span) and complex (e.g., operation span) working memory processing and the degree of interference from irrelevant sound (e.g., Beaman, 2004; Ellermeier \& Zimmer, 1997; Elliott \& Cowan, 2005). As such, the functional character of auditory distraction described here differs from that reported elsewhere, where individual differences in distraction are related to working memory capacity (e.g., Vogel, McCollough, \& Machizawa, 2005). This mode of distraction therefore poses a challenge to the traditional conceptualization of the processes involved in the ability to select relevant information in the presence of the irrelevant.

In this article, we test the novel hypothesis that distraction in this setting, rather than being a negative function of executive resources, is in fact a positive function of the efficacy of obligatory perceptual organization of auditory sequences. Our emphasis on disruption due to similarity of process, described above, leads us to propose that it is the facility with which auditory sequences are perceptually organized that determines how disruptive such sequences will be when they occur as irrelevant sound. On the face of it, this might appear to have already been ruled out by the data, since there is no relationship between serial recall performance (a measure of sequential processing) and the extent to which that performance is susceptible to disruption (Ellermeier \& Zimmer, 1997; Elliott \& Cowan, 2005). However, serial recall is a measure of deliberate sequence processing, whereas irrelevant sound, by definition, is not deliberately processed. As such, what is needed to test this hypothesis is a task that measures the efficacy of obligatory (i.e., nondeliberate) auditory perceptual organization. We describe below two versions of a simple sequenceprocessing task; one requires deliberate recoding, and the other, we argue (and demonstrate), may be accomplished on the basis of obligatory, global processing. We then relate individual differences in performance on these two sequence-processing tasks to individual differences in both serial recall performance and individual differences in the susceptibility of that performance to distraction by irrelevant sound.

\section{GENERAL METHOD}

\section{Design and Procedure}

Two types of auditory sequence-processing tasks were employed in the study (based on those used by Foxton et al., 2003). Pure tones were synthesized in an atonal scale spanning the octave starting at $250 \mathrm{~Hz}$, divided into seven equally spaced logarithmic steps. Each tone was $250 \mathrm{msec}$ in length, with rise and fall times of $20 \mathrm{msec}$. Tones were assembled into pairs of six-tone sequences on which participants had to make same/different judgments. Each sequence was 1,500 msec in length, and the standard and comparison sequences were separated by $1,000 \mathrm{msec}$ of silence. The two types of task were as follows. In one, standard and comparison sequences were identical on "same" trials, whereas on "different" trials one of the tones, other than the first and last, was changed by two steps either up or down in frequency. In the second task, "same" trials involved raising or lowering all tones between the standard and comparison sequences while maintaining the same overall "melody" in the sequence. The "different" trials in this version of the task also involved the same transposition of the sequence between standard and comparison, but this time with an additional two-step change to one (other than the first or last) of the tones such that the comparison sequence no longer carried the same melody as the standard (see Figure 1).

We argue that the former version may be accomplished on the basis of global pattern matching, without the need for deliberate recoding of the sequence (e.g., Warren, 1999), whereas the latter version requires deliberate recoding to enable comparison of the "melody" across different frequency ranges. Part I of the experiment establishes the empirical evidence for this characterization. 


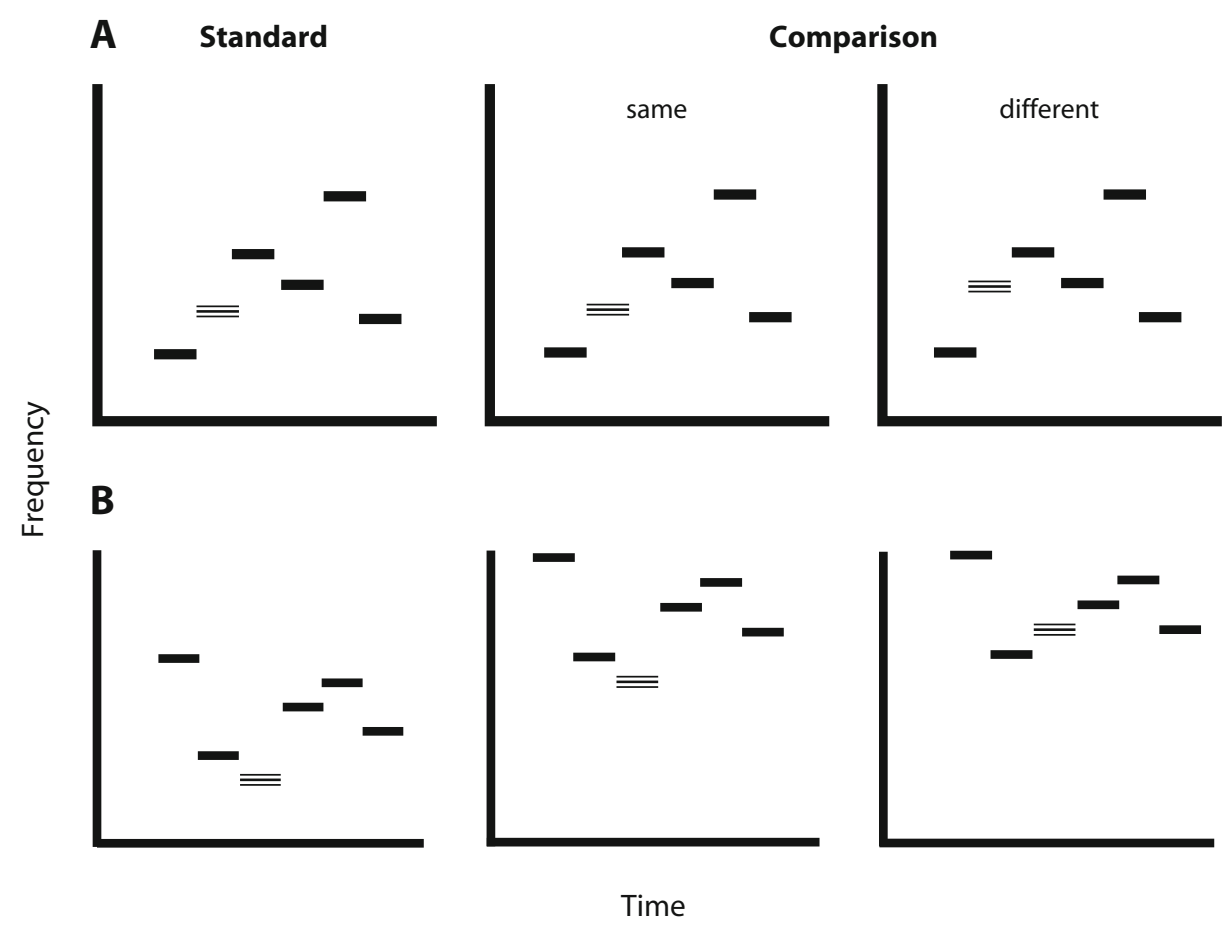

Figure 1. Schematic illustration of the tasks used in Part I. (A) An example of same and different global pattern-matching stimuli. (B) An example of the deliberate recoding stimuli.

\section{Part I}

\section{Method}

Fifty participants recruited from the Cardiff School of Psychology undergraduate population received course credit for participation and made same/different judgments on 32 pairs of sequences in the deliberate, recoding version of the task and 32 pairs in the global patternmatching version. Sixteen of each type of judgment were made under control conditions, and 16 while whispering the letter sounds $x, y$, and $z$ repeatedly at a rate of $1 \mathrm{cycle} / \mathrm{sec}$. We have shown elsewhere that such articulatory suppression inhibits the ability to recode sequential information (e.g., Macken \& Jones, 1995) without impeding certain aspects of auditory perceptual processing (Jones, Macken, \& Nicholls, 2004). Participants were seated in a sound-attenuated booth, and stimuli were presented over Sennheiser headphones, with participants making their same/different responses via a computer keyboard. The procedure was self-paced, with participants initiating each trial by pressing the mouse button. Participants performing close to chance on one task and close to ceiling on the other were excluded in order to avoid the possibility that any dissociation in performance across the two tasks could be attributed merely to floor or ceiling effects in one or the other. This led to the exclusion of 13 participants, all of whom performed at chance on the deliberate recoding task under control conditions, while performing above $90 \%$ on the global pattern-matching task.

\section{Results}

Mean number of correct judgments in a repeated measures 2 (sequence-processing task: deliberate recoding vs. global pattern matching) $\times 2$ (condition: control vs. suppression) ANOVA revealed main effects of both task and condition $\left[F(1,36)=31.25, M S_{\mathrm{e}}=44.40, p<.0001\right.$, and $F(1,36)=117.55, M S_{\mathrm{e}}=80.62, p<.0001$, respectively]. Critically, however, these two factors interacted $[F(1,36)=$ $\left.18.28, M S_{\mathrm{e}}=41.33, p<.0001\right]$, such that, whereas performance in the two tasks under control conditions was broadly similar $(72.46 \%$ and $67.58 \%$ in the global pattern-matching and deliberate recoding tasks, respectively) $[F(1,36)=$ $2.35, p=.13, d=0.22]$, under suppression, performance on the recoding task was significantly worse than on the global task (50.34\% and $60.98 \%$, respectively) $[F(1,36)=$ $31.99, p<.0001, d=1.09]$. Indeed, deliberate recoding performance was at chance level $(50 \%)$ under suppression $[t(36)=0.25, p>.10]$, whereas the global patternmatching version of the task, although being impaired under suppression relative to control, was still performed significantly above chance $[t(36)=9.63, p<.01]$.

Above-chance performance in global sequenceprocessing where recoding is prevented supports our claim that this task may be accomplished on the basis of perceptual processes, independent (at least in part) of deliberate recoding mechanisms used in the other version of the task. These tasks, therefore, enable us to assess the hypothesis that whereas sequence processing based on obligatory global pattern matching should predict disruptibility, deliberate sequence-processing processes will not.

\section{Part II}

\section{Method}

Thirty-two of the undergraduates who participated in Part I returned to perform an irrelevant sound experiment that involved serial recall of 36 lists of random orderings of the digits 1 to 8 . Memory items were presented individually on the computer screen at a rate of $1 / \mathrm{sec}$ followed by a 10 -sec retention interval, followed by serial recall. Participants initiated each trial by means of the mouse button. On 18 of the trials, distributed randomly throughout the procedure, a continuous looping of precisely the type of tone sequences presented in Part I was presented via headphones during presentation and retention intervals. Participants were instructed to ignore the sound and were assured that they would not be asked about it. Correct recall averaged 
Table 1

Descriptive Statistics and Correlation Matrix for Four Variables

\begin{tabular}{lcccccccccrr}
\hline & & & & & & & \multicolumn{3}{c}{ Kurtosis } & \multicolumn{2}{c}{ Skew } \\
\hline DR & DR & GP-M & SR & D & Mean & SD & $M$ & $S E$ & & $M$ & $S E$ \\
GP-M & - & $.62^{* *}$ & $.40^{*}$ & $.44^{*}$ & $67.58 \%$ & 10.79 & -.57 & .81 & & .21 & .41 \\
SR & & - & .13 & $.57^{* *}$ & $72.46 \%$ & 10.54 & -.47 & .81 & -.44 & .41 \\
D & & & - & .01 & $14.66 / 18$ & 1.83 & -.75 & .81 & & .32 & .41 \\
\hline
\end{tabular}

Note-DR, performance on the deliberate recoding task; GP-M, performance on the global pattern-matching task; SR, performance on the serial recall task under quiet conditions; $\mathrm{D}$, the degree of disruptibility due to irrelevant sound (performance in quiet minus performance in sound). $\quad{ }^{*} p<.05 . \quad{ }^{* *} p<.01$.

across serial position was then calculated for each participant in quiet and sound conditions, and degree of disruptibility for each participant was calculated as the difference between performance in quiet and performance in sound (see Ellermeier \& Zimmer, 1997). Correla-

\section{A}

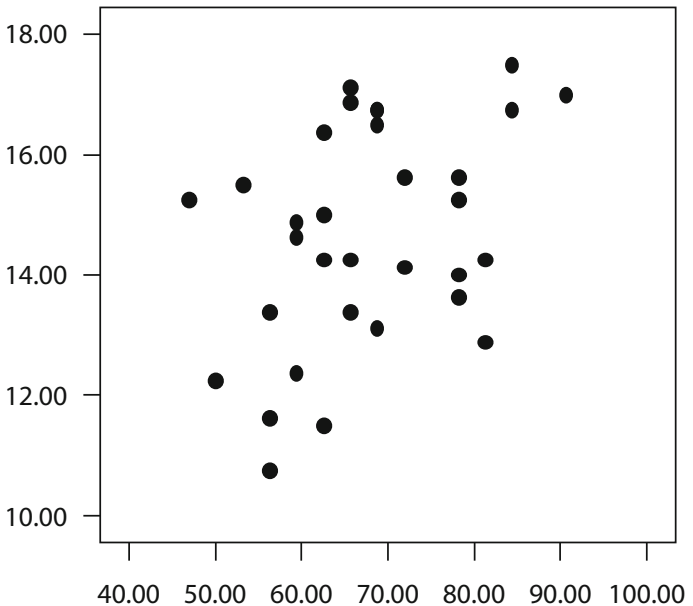

Deliberate Recoding Performance (\%)

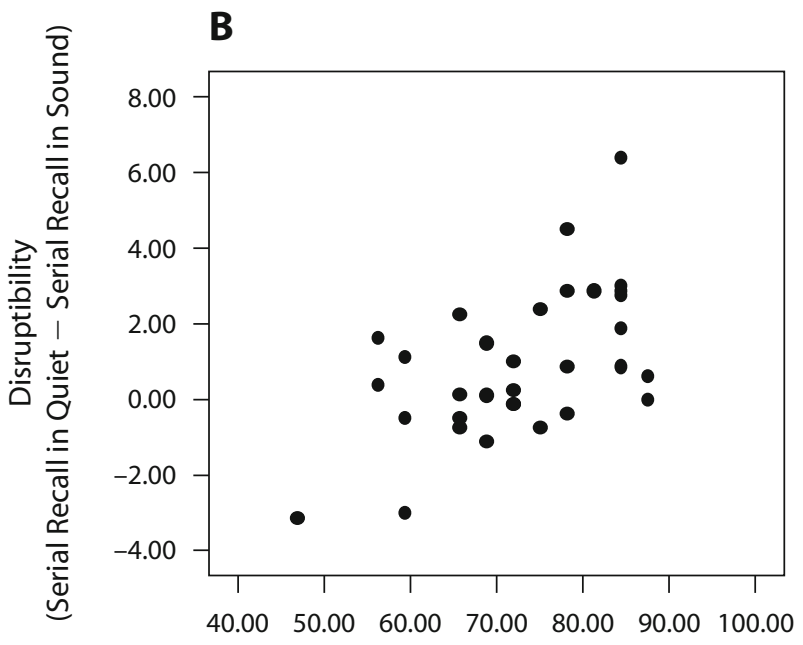

Global Pattern-Matching Performance (\%)

Figure 2. Scatterplots of the two key positive relationships. (A) Deliberate recoding performance and serial recall performance. (B) Global pattern-matching performance and disruptibility. tions between these variables and those from Part I (i.e., global pattern matching and deliberate recoding performance), along with relevant descriptive statistics, are presented in Table 1.

\section{Results}

The two dependent variables, disruptibility and serial recall performance (in quiet), were each entered into regression analyses with the two predictor variables from Part Inamely, performance on the deliberate sequence-processing task and performance on the global sequence-processing task (both measures taken from the control conditions of Part I). As can be seen from this analysis (see Table 2), performance on the deliberate recoding task is significantly and positively related to performance on the serial recall task; better performance on one is associated with better performance on the other (see Figure 2A). This is to be expected, given that both tasks require some recoding of the initial input: to enable rehearsal and reproduction of the sequence in the case of serial recall, and to enable comparison across frequency ranges in the case of the deliberate sequence-processing task. However, performance on this deliberate sequenceprocessing task did not significantly predict the degree of disruptibility (as was also the case for serial recall performance; see also Ellermeier \& Zimmer, 1997, and Elliott \& Cowan, 2005, for the same finding). But, in accordance with our hypothesis, performance on the global pattern-matching task did predict the degree of disruptibility due to the irrelevant sound. This indicates that better performance in global auditory sequence processing is related to greater disruption to serial recall performance in the presence of to-be-ignored, task-irrelevant auditory sequences (see Figure 2B). In other words, the greater the degree of facility in processing auditory sequences in a setting where deliberate recoding need not be engaged, the more susceptible an individual is to the interfering effects of irrelevant sound. Confirming the independence of such global sequence processing from that required for serial recall, the relationship between performance on the global task and the serial recall task was nonsignificant (see Table 1). This finding adds further weight to our characterization of this task as reflecting perceptual, rather than memorial, processes, since performance on it is unrelated to even relatively simple measures of storage capacity (i.e., serial recall; see Vogel et al., 2005).

\section{GENERAL DISCUSSION}

The results of this study show that individual differences in susceptibility to disruption by task-irrelevant, 
Table 2

Results of Regression Analyses for Two Predictor Variables and Two Dependent Variables

\begin{tabular}{cc}
\hline Predictor & $\beta$ \\
\hline Global Pattern Matching & \\
Serial recall & -.19 \\
Disruptibility & $.47^{*}$ \\
Deliberate Recoding & \\
Serial recall & $.52^{*}$ \\
Disruptibility & .15 \\
\hline
\end{tabular}

${ }^{*} p<.05$.

background sound are related to individual differences in processes involved with the global processing of auditory sequences. Furthermore, those processes that are engaged when a sequence-processing task requires deliberate recoding of the perceptual input do not predict disruptibility, although they are related to other, deliberate, sequencing processes (i.e., serial recall).

These findings contribute to a coherent account of why previous attempts to predict distractibility proved unsuccessful; to the extent that individual differences in deliberate sequence processing, as is necessary in serial recall as well as in the more executive-laden span tasks, have been examined, no relation between them and disruptibility has been found. In one sense, this should not be surprising. Since the irrelevant sound is not processed deliberately, factors associated with deliberate processing might not be expected to be important. On the other hand, since resistance to distraction is usually attributed to the type of deliberate, executive/ working memory processes involved in performing complex span tasks (Conway et al., 2001; Engle et al., 1999), a relationship to individual differences in susceptibility to irrelevant sound would be predicted. However, as has now been demonstrated elsewhere several times (Beaman, 2004; Ellermeier \& Zimmer, 1997; Elliott \& Cowan, 2005), this is not borne out by the data. Furthermore, although measures of more "simple" working memory capacity (e.g., storage capacity in terms of number of items held in working memory) have also been shown to be related to measures of distractibility in other settings (e.g., Vogel et al., 2005), this is clearly not the case in the present setting. First, since serial recall performance itself is unrelated to distractibility, clearly working memory capacity as measured by such a task is not related to the disruptive effect of irrelevant sound. Furthermore, although performance on the global pattern-matching task is related to distractibility in our study, this is unlikely to be because performance in such a task is simply another measure of such capacity, since it, too, is unrelated to capacity, as measured by serial recall. Finally, and perhaps most compellingly, although accounts of distractibility in terms of capacity (however that may be conceived) predict a negative relationship between capacity and distractibility (i.e., higher capacity is associated with lower distractibility), what we have demonstrated here is a positive relationship between performance on the global task and distractibility (i.e., better global sequence processing is associated with greater distractibility). It is hard indeed to see how these more traditional accounts of selective attention and distractibility (e.g., Cowan, 1995) could be made to account for both negative and positive relationships between performance and such distractibility. As such, we argue (as we have elsewhere; e.g., Jones et al., 1997; Macken, Tremblay, Alford, \& Jones, 1999) that the pervasive mode of distraction represented by the irrelevant sound effect cannot be accounted for in such terms.

These results do fit with the account of the effect of task-irrelevant sound we have presented elsewhere. As described above, we argue that those processes involved in the preattentive, obligatory organization of the auditory environment underpin distraction within the present paradigm, and the functional character of this distraction is well described as being due to interference based on similarity of process: The obligatory processing of irrelevant auditory sequences leads to interference with the deliberate processing of memory sequences. This is not to say that inhibitory processes associated with working memory capacity are not engaged within this experimental setting. For example, a to-be-ignored sequence of auditory digits on a given trial is less well remembered when the same sequence of digits is presented visually as a to-be-remembered list on a subsequent trial than an unrelated sequence of the same digits (Hughes \& Jones, 2003b). This suggests that there are sequence-specific inhibitory processes applied to the to-be-ignored input, leading to negative priming of that sequence when it becomes a candidate for deliberate processing at a later stage. Importantly, however, the efficacy of that inhibitory process will not determine the disruptive potency of that to-be-ignored sequence; the damage, as it were, has already been done, by mere virtue of the irrelevant sequence entering the processing stream.

We argue therefore that the effect of task-irrelevant sound, rather than being a manifestation of mechanisms associated with the executive control of attention, reflects the obligatory processing of sound within a system involved with auditory-motor integration (e.g., Hickok \& Poeppel, 2000,2004 ). The functional nature of the irrelevant sound effect implies that the mere presence of sequences of sound impedes our ability to engage in the deliberate rehearsal involved in performing short-term serial recall (importantly in this respect, when subvocal rehearsal is prevented during serial recall, irrelevant sound ceases to have its disruptive effect; Jones et al., 2004). Therefore, there must be some point in the processing stream at which the memory sequence and the auditory sequence come into conflict. Such a system cannot be the phonological store of working memory (e.g., Baddeley, 1990; Salamé \& Baddeley, 1982), since not only do the phonological characteristics of the sound have no impact on its disruptive potency (e.g., Jones \& Macken, 1995a, 1995b), the sound need not possess any phonological content whatsoever in order for disruption to occur (e.g., Jones \& Macken, 1993). Rather, we argue that the irrelevant sound effect reflects the obligatory processing of sound within, not a phonological working memory system per se, but a system involved in general auditorymotor integration that is co-opted to perform short-term memory tasks (see also Hickok \& Poeppel, 2004; Macken \& Jones, 1995; Wilson, 2001; Wilson \& Fox, 2007). That neural systems associated with performance of verbal short-term memory tasks also appear to be involved in both 
active and passive listening to verbal and nonverbal auditory material lends weight to this possibility (e.g., Hickok, Buchsbaum, Humphries, \& Muftuler, 2003).

In conclusion, we have argued here for a novel pattern of auditory distraction that cannot be accounted for simply within the account of selection and inhibition normally invoked to explain such distraction. Rather, we see distraction as being a positive reflection of the operation of obligatory auditory-motor mapping systems, which, although clearly embodying adaptive mechanisms for learning via observation and even imitation (see, e.g., Doupe \& Kuhl, 1999; Heyes, 2001), necessarily give rise to representations that compete for access to systems required for control of goal-directed behavior; auditory distraction is caused by the efficacy with which these auditory-motor mapping systems obligatorily derive such representations from auditory sequences.

\section{AUTHOR NOTE}

The research for this article was supported by the Economic and Social Research Council of the U.K. (Award RES-062-23-0011). The authors thank Rob Hughes for useful discussion and comments on an earlier draft. Correspondence concerning this article should be addressed to W. J. Macken, School of Psychology, Cardiff University, Cardiff CF10 3AT, Wales (e-mail: macken@cardiff.ac.uk).

\section{REFERENCES}

Baddeley, A. D. (1990). Human memory: Theory and practice. Needham Heights, MA: Allyn \& Bacon.

Banbury, S., \& Berry, D. (1998). Disruption of office-related tasks by speech and office noise. British Journal of Psychology, 893, 499-517.

BEAMAN, C. P. (2004). The irrelevant sound phenomenon revisited: What role for working memory capacity? Journal of Experimental Psychology: Learning, Memory, \& Cognition, 30, 1106-1118.

BEAMAN, C. P., \& JoNES, D. M. (1997). Role of serial order in the irrelevant speech effect: Tests of the changing-state hypothesis. Journal of Experimental Psychology: Learning, Memory, \& Cognition, 23, 459-471.

Colle, H. A., \& Welsh, A. (1976). Acoustic masking in primary memory. Journal of Verbal Learning \& Verbal Behavior, 15, 17-32.

Conway, A. R. A., Cowan, N., \& Bunting, M. F. (2001). The cocktail party phenomenon revisited: The importance of working memory capacity. Psychonomic Bulletin \& Review, 8, 331-335.

Cowan, N. (1995). Attention and memory: An integrated framework. New York: Oxford University Press.

Daneman, M., \& Carpenter, P. A. (1980). Individual differences in working memory and reading. Journal of Verbal Learning \& Verbal Behavior, 19, 450-466.

Doupe, A. J., \& KuHL, P. (1999). Birdsong and human speech: Common themes and mechanisms. Annual Review of Neuroscience, 22, 567-631.

Ellermeier, W., \& Zimmer, K. (1997). Individual differences in susceptibility to the "irrelevant speech effect." Journal of the Acoustical Society of America, 102, 2191-2199.

Elliott, E., \& Cowan, N. (2005). Coherence of the irrelevant-sound effect: Individual profiles of short-term memory and susceptibility to task-irrelevant materials. Memory \& Cognition, 33, 664-675.

Engle, R., Kane, M., \& Tuholski, S. (1999). Individual differences in working memory capacity and what they tell us about controlled attention, general fluid intelligence, and functions of the prefrontal cortex. In A. Miyake \& P. Shah (Eds.), Models of working memory: Mechanisms of active maintenance and executive control (pp. 102134). Cambridge: Cambridge University Press.

Engle, R., Tuholski, S., Laughlin, J., \& Conway, A. R. A. (1999). Working memory, short-term memory, and general fluid intelligence: A latent variable approach. Journal of Experimental Psychology: General, 128, 309-331.

Foxton, J. M., Talcott, J. B., Witton, C., Brace, H., McIntyre, F.,
\& GRIFFITHS, T. (2003). Reading skills are related to global, but not local, acoustic pattern perception. Nature Neuroscience, 6, 343-344.

Hellbrück, J., Namba, S., \& Kuwano, S. (1996). Irrelevant background speech and human performance: Is there long-term habituation? Journal of the Acoustical Society of Japan, 17, 239-247.

Heyes, C. (2001). Causes and consequences of imitation. Trends in Cognitive Sciences, 5, 253-261

Hickok, G., Buchsbaum, B., Humphries, C., \& Muftuler, T. (2003). Auditory-motor interaction revealed by fMRI: Speech, music, and working memory in area Spt. Journal of Cognitive Neuroscience, 15, 673-682.

HickoK, G., \& PoepPeL, D. (2000). Towards a functional neuroanatomy of speech perception. Trends in Cognitive Sciences, 4, 131-138.

HickoK, G., \& PoEPPEL, D. (2004). Dorsal and ventral streams: A framework for understanding aspects of the functional anatomy of language. Cognition, 92, 67-99.

Hughes, R. W., \& Jones, D. M. (2003a). Indispensable benefits and unavoidable costs of irrelevant sound for cognitive functioning. Noise \& Health, 6, 63-76.

Hughes, R. W., \& Jones, D. M. (2003b). A negative order-repetition priming effect: Inhibition of order in unattended auditory sequences? Journal of Experimental Psychology: Human Perception \& Performance, 29, 199-218.

JoNes, D. M., \& MACKEN, W. J. (1993). Irrelevant tones produce an irrelevant speech effect: Implications for phonological coding in working memory. Journal of Experimental Psychology: Learning, Memory, \& Cognition, 19, 369-381.

Jones, D. M., \& MACKEN, W. J. (1995a). Organizational factors in the effect of irrelevant speech: The role of spatial location and timing. Memory \& Cognition, 23, 192-200.

Jones, D. M., \& Macken, W. J. (1995b). Phonological similarity in the irrelevant speech effect: Within- or between-stream similarity? Journal of Experimental Psychology: Learning, Memory, \& Cognition, 21, 103-115

Jones, D. M., Macken, W. J., \& Mosdell, N. (1997). The role of habituation in the disruption of recall by irrelevant sound. British Journal of Psychology, 88, 549-564.

Jones, D. M., Macken, W. J., \& Nicholls, A. (2004). The phonological store of working memory: Is it phonological and is it a store? Journal of Experimental Psychology: Learning, Memory, \& Cognition, 30, 656-674.

Lavie, N. (2005). Distracted and confused? Selective attention under load. Trends in Cognitive Sciences, 9, 75-82.

MACKEN, W. J., \& JONES, D. M. (1995). Functional characteristics of the inner voice and the inner ear: Single or double agency? Journal of Experimental Psychology: Learning, Memory, \& Cognition, 21, 436-448.

Macken, W. J., Tremblay, S., Alford, D., \& Jones, D. M. (1999). Attentional selectivity in short-term memory: Similarity of process, not similarity of content, determines disruption. International Journal of Psychology, 34, 322-327.

Salamé, P., \& Baddeley, A. [D.] (1982). Disruption of short-term memory by unattended speech: Implications for the structure of working memory. Journal of Verbal Learning \& Verbal Behavior, 21, 150-164.

Shiffrin, R. M., \& Schneider, W. (1977). Controlled and automatic human information processing: II. Perceptual learning, automatic attending and a general theory. Psychological Review, 84, 127-190.

Süß, H.-M., Oberauer, K., Wittmann, W., Wilhelm, O., \& Schulze, R. (2002). Working-memory capacity explains reasoning ability - and a little bit more. Intelligence, 30, 261-288.

Vogel, E. K., McCollough, A. W., \& Machizawa, M. G. (2005). Neural measures reveal individual differences in controlling access to working memory. Nature, 438, 500-503.

WARREN, R. (1999). Auditory perception: A new analysis and synthesis. New York: Cambridge University Press.

WILSON, M. (2001). The case for sensorimotor coding in working memory. Psychonomic Bulletin \& Review, 8, 44-57.

WiLson, M., \& Fox, G. (2007). Working memory for language is not special: Evidence for an articulatory loop for novel stimuli. Psychonomic Bulletin \& Review, 14, 470-473.

(Manuscript received April 20, 2007; revision accepted for publication August 1, 2008.) 